\title{
A CMOS 256-pixel Photovoltaics-powered Implantable Chip with Active Pixel Sensors and Iridium-oxide Electrodes for Subretinal Prostheses
}

\author{
Chung-Yu Wu, ${ }^{1 *}$ Po-Han Kuo, ${ }^{1}$ Po-Kang Lin, ${ }^{2,3}$ Po-Chun Chen, ${ }^{4}$ \\ Wei-Jie Sung, ${ }^{1}$ Jun Ohta, ${ }^{5}$ Takashi Tokuda, ${ }^{5}$ and Toshihiko Noda ${ }^{5}$ \\ ${ }^{1}$ Department of Electrical Engineering, National Chiao Tung University, \\ 1001 University Road, Hsinchu City 30010, Taiwan \\ ${ }^{2}$ Department of Ophthalmology, Taipei Veterans General Hospital, \\ 201, Sec. 2, Shih-Pai Rd., Taipei 10608, Taiwan \\ ${ }^{3}$ School of Medicine, National Yang-Ming University, \\ 155, Sec. 2, Linong St., Taipei 11221, Taiwan \\ ${ }^{4}$ Department of Materials and Mineral Resources Engineering, National Taipei University of Technology, \\ 1, Sec. 3, Zhongxiao E. Rd., Taipei 10608, Taiwan \\ ${ }^{5}$ Graduate School of Materials Science, Nara Institute of Science and Technology, \\ 8916-5 Takayama, Ikoma, Nara 630-0101, Japan
}

(Received July 14, 2017; accepted September 6, 2017)

Keywords: divisional power supply scheme, active pixel sensor, photovoltaic cell, CMOS image sensor, biocompatible package

A CMOS implantable chip with 256 active pixel sensors (APSs), on-chip photovoltaic cells, and iridium-oxide ( $\mathrm{IrOx}$ ) electrodes is proposed and designed for subretinal prostheses. In the proposed chip, the on-chip electrode surface is deposited with IrOx by RF sputtering and photolithography patterning. The divisional power supply scheme (DPSS) is adopted to generate sufficient stimulation current whereas the APS is used to enhance the image sensitivity. The proposed chip consists of a $16 \times 16$ photodiode array with 8 DPSS divisions, control signal generator circuits, and photovoltaic cells. It is designed and fabricated into $180 \mathrm{~nm}$ CMOS image sensor (CIS) technology and postprocessed with an IrOx coating. From the electrical measurement results, the fabricated chip has a peak output stimulation current of $16.7 \mu \mathrm{A}$ under the equivalent electrode impedance load. The stimulation frequency is $30.2 \mathrm{~Hz}$ and the amount of injected charges at each pixel is $3.5 \mathrm{nC}$. Both image light sensitivity and injection charges are significantly improved. The surface morphology, crystallinity, charge storage capacity, and biocompatibility of sputtering iridium oxide film (SIROF) were investigated. As a result, the SIROF has desirable physical and electrochemical properties that make it suitable for the neurostimulation electrodes on the 256-pixel implantable chip. The 7-month biocompatibility and charge delivery capability of the fabricated chip have been confirmed by electroretinography (ERG) measurement on a Lanyu minipig in in vivo animal tests.

*Corresponding author: e-mail: peterwu@mail.nctu.edu.tw http://dx.doi.org/10.18494/SAM.2018.1664 


\section{Introduction}

Retinitis pigmentosa (RP) and age-related macular degeneration (AMD) are two diseases in which degenerated photoreceptors fail to transfer visual information to other retinal cells in the retinal network. As a result, the patients lose their vision in the late stage. A promising way to restore partial vision for these patients is to implant retinal prosthetic devices and apply electrical stimulation to ganglion cells or other retinal cells so that visual signals can be transmitted to the visual cortex.

Among all proposed retinal prostheses, subretinal and epiretinal implants are of great interest and many structures have recently been proposed. ${ }^{(1-15)}$ The stimulating electrodes of a subretinal implant are positioned under photoreceptors whereas those of an epiretinal implant are positioned above ganglion cells. In the subretinal implants, ${ }^{(8-15)}$ passive photodiode array, ${ }^{(9)}$ active photodiode sensors with current amplifiers, ${ }^{(8,11,12)}$ or active pixel circuits, ${ }^{(10,13)}$ have been proposed. Most subretinal implants require a wire through the eyeball to provide power supply. ${ }^{(13,14)}$ A wirelessly powered subretinal implant is designed with a subdermal coil embedded behind the ear. ${ }^{(15)}$ In Refs. 9, 11, and 12, the optical powering scheme was proposed and the incident light was converted to stimulation currents by photodiodes. Compared with the RF powering schemes, no external battery, direct wire, or RF connection is required. Therefore, the operation wound and surgery complexity can be reduced. However, biphasic current stimulation is not realizable and charge balancing is not guaranteed.

In Ref. 16, a $16 \times 16$ self-powered implantable chip was proposed. In the proposed structure, the active pixel sensor (APS) and on-chip photovoltaic cells in CMOS image sensor (CIS) process technology are adopted to increase both light sensitivity and photovoltaic power generation efficiency. The divisional power supply scheme (DPSS) is also used to decrease the overall power consumption during stimulation and increase the power usage efficiency.

The electrode materials used in the implantable neurostimulation systems can be divided into two distinct categories on the basis of the charge transfer mechanism at the electrode interface: (1) capacitive charge injection materials ${ }^{(17-19)}$ and (2) faradaic charge injection materials. ${ }^{(20)}$ In general, electrostimulation by the faradaic mechanism brings about better charge injection performance than that by the capacitive mechanism. Among various electrode materials, iridium oxide ( $\mathrm{IrOx}$ ), a functional ceramic material, can adequately provide the required charges for stimulation through electrochemical reduction and oxidation reactions at the electrode interface without damaging surrounding tissues. ${ }^{(20)}$ As a result, advantages such as high charge injection capability, high safety voltage, good stability, and high biocompatibility render IrOx the most promising material for implantable neurostimulation electrodes.

It is known that electrode performance can be improved by applying a surface coating of a high-performance material, and IrOx is the leading candidate for such a material. ${ }^{(21-25)}$ An IrOx surface coating layer can be formed by several techniques. Among them, IrOx deposited by reactive sputtering has the advantage of high controllability of the film properties by regulating the sputtering conditions. 
In this paper, an improved design of the 256-pixel implantable chip is proposed. In the improved design, the on-chip electrode surface is deposited with IrOx by RF sputtering and photolithography patterning. Moreover, the integration capacitance at APS is decreased to increase the light sensitivity. The integrated voltage of APS is sent out through an operational amplifier instead of a simple source follower to reduce the threshold voltage drop and increase the dynamic range. The architecture and circuits of the implantable chip are described in Sect. 2. The IrOx process on electrodes and measurement results are presented in Sect. 3. In Sect. 4, electrical test and in vivo test results are described. In the last section, the conclusions are drawn.

\section{Chip Architecture and Circuits}

\subsection{On-chip photovoltaic cells}

The layout and cross-sectional views of the on-chip photovoltaic-cell structure with integrated N-channel MOS (NMOS) and P-channel MOS (PMOS) devices are shown in Fig. 1, where a twin-well CMOS CIS technology with a deep N-well (DNW) structure was adopted. The reverse-biased $\mathrm{N}+/ \mathrm{P}$-well junction with a grounded $\mathrm{P}$-well and surrounding $\mathrm{N}$-well/DNW is adopted as the photodiode sensor, whereas all $\mathrm{N}+/ \mathrm{P}$-well, $\mathrm{P}$-well/N-well, and $\mathrm{P}$-well/DNW junctions are connected in parallel to form a photovoltaic cell. In the photovoltaic cell, the $\mathrm{P}$-well anode is the power supply $V_{D D}$ while the $\mathrm{N}+/ \mathrm{N}$-well/ DNW cathode is the ground (GND). The power supply $V_{D D}$ is about $0.5 \mathrm{~V}$. Since the $\mathrm{P}$-substrate is kept floating and the N-well/DNW is grounded, the unwanted photocurrent leakage can be avoided. The NMOS transistor in a P-well connected to GND is placed in a DNW connected to $V_{D D}$ so that its $\mathrm{P}$-well body can be isolated from the P-substrate. The PMOS transistor is connected similarly to that in the conventional structure where the N-well body is connected to the highest voltage $V_{D D}$ of the system.

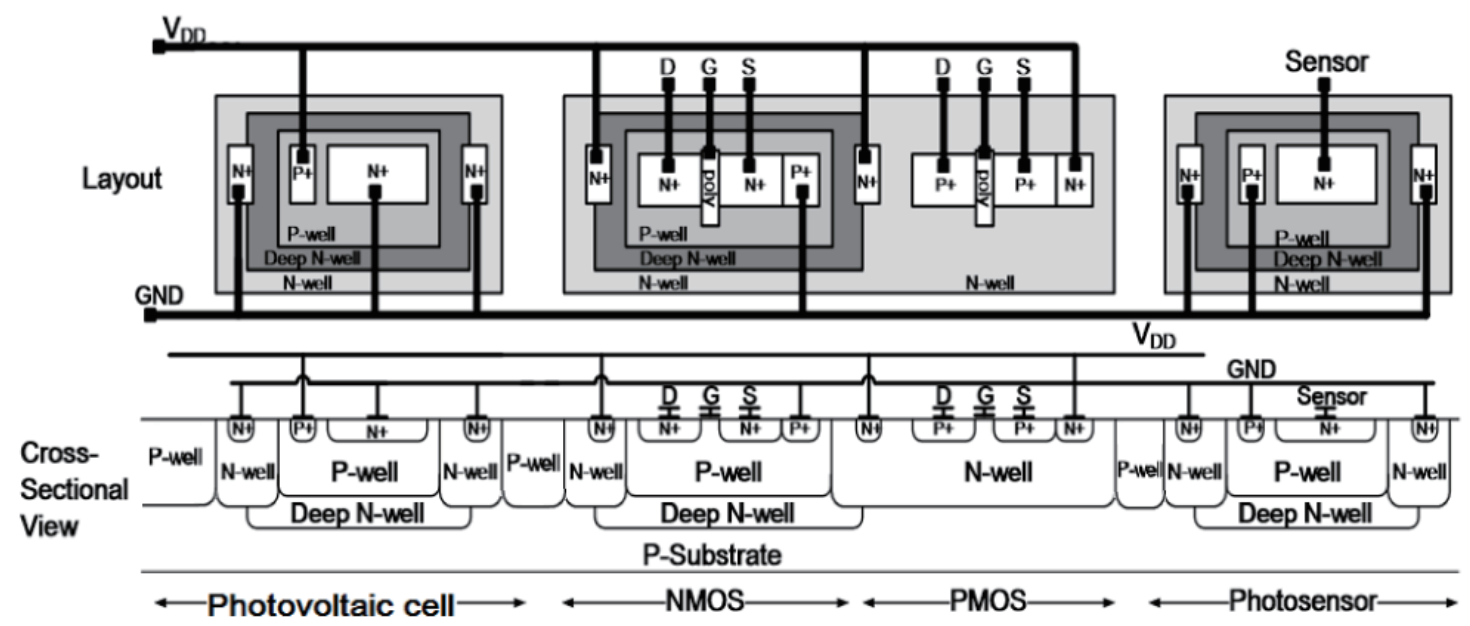

Fig. 1. Layout and cross-sectional view of the proposed photovoltaic cell structure integrated with NMOS and PMOS devices on the same chip. 
The layout size of a single photovoltaic cell is $5 \times 5 \mu \mathrm{m}^{2}$. There is a total of 150769 photovoltaic cells designed on this chip. In the adopted CMOS CIS technology, the silicide process is used to produce a nontransparent silicide layer. Thus, one more mask is needed to block the silicide in the photosensing regions.

\subsection{DPSS}

It is known that an afterimage can persist approximately $40 \mathrm{~ms}$ in the human visual system. This phenomenon is called the persistence of vision. ${ }^{(26)}$ On the basis of this phenomenon, the DPSS is adopted to increase the efficiency of photovoltaic power supply usage and increase the stimulation currents. In the DPSS, as shown in Fig. 2, the 256-pixel array is further divided into eight groups that are powered by all photovoltaic cells alternately through a control clock of $30.2 \mathrm{~Hz}$. In one clock period, only the pixels of one group are powered by all onchip photovoltaic cells to generate the positive stimulation currents. In the next period, those of the same group are powered to generate the negative stimulation currents. Thus biphasic stimulation can be realized. If the pixels of eight groups are stimulated alternately within the time of vision persistence $(40 \mathrm{~ms})$, a complete image can be sensed. With the DPSS, the stimulation current could be eight times higher than that when all groups of pixels are powered simultaneously.

\subsection{Pixel array and clock generator}

The schematic of the pixel array and control-signal generator with the proposed DPSS is shown in Fig. 3. The 256-pixel array is divided into eight groups with 32 pixels each, as marked by numbers in the pixel array shown in Fig. 3(b). As shown in Fig. 3(a), the control signal generator consists of a CMOS ring oscillator, frequency dividers, and combinational logic circuits. The clock frequency is set to $61.85 \mathrm{kHz}$ so that the requirement of vision persistence can be satisfied with the DPSS. The control signal generator generates nonoverlapping eight-phase signals $V_{P H 1}-V_{P H 8}$ that control switches in the 1st-8th groups.

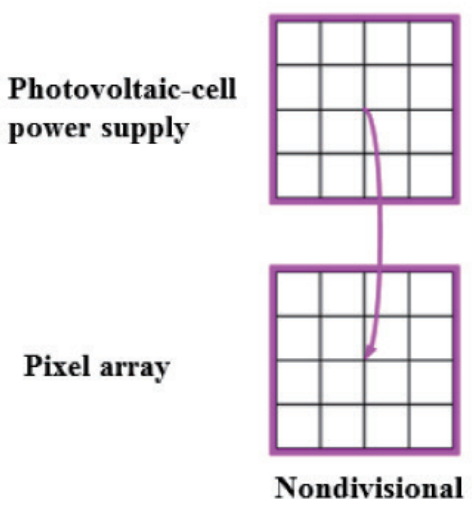

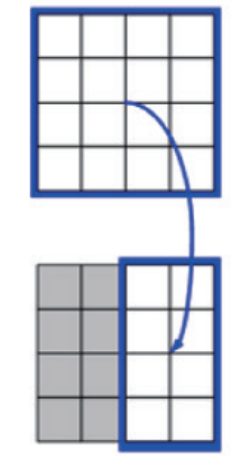

Two-block division

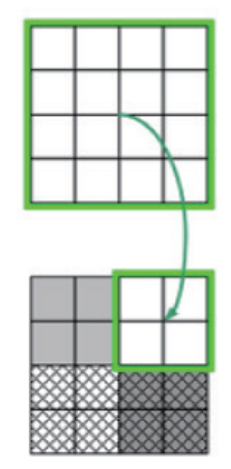

Four-block division

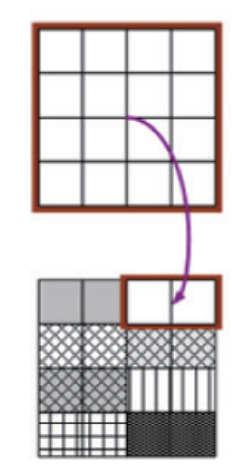

Eight-block division

Fig. 2. (Color online) Illustration of DPSS. 


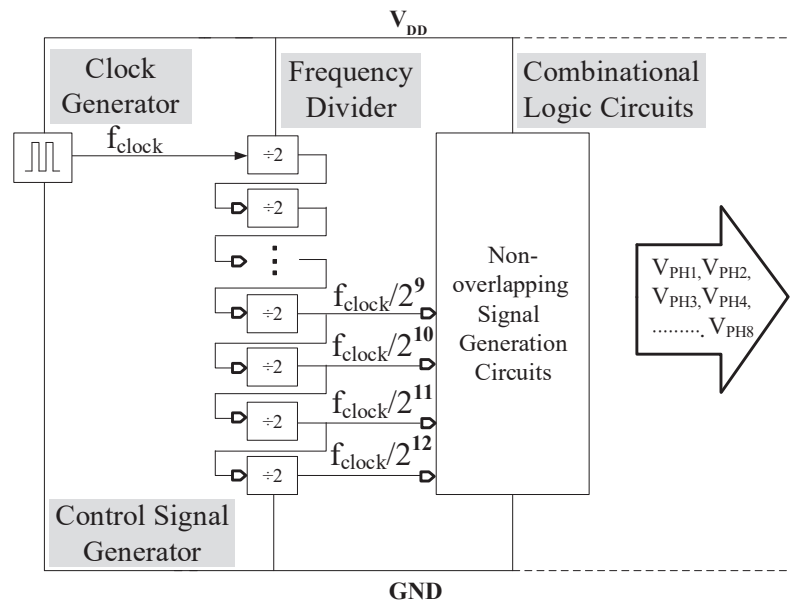

(a)

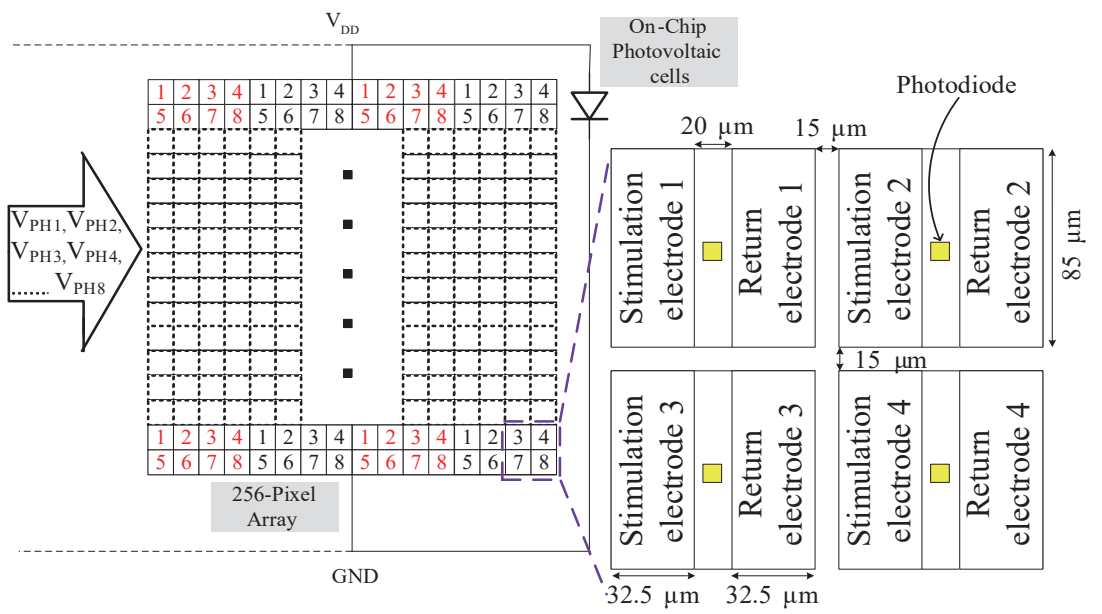

(b)

Fig. 3. (Color online) Schematic of (a) control-signal generator and (b) 256-pixel array with the DPSS. The control signal generator generates nonoverlapping eight-phase signals $V_{P H 1}-V_{P H 8}$ that control the switches in the 1st-8th pixel groups from the $61.85 \mathrm{kHz}$ clock signal $f_{\text {clock }}$.

In each pixel, the photodiode and APS circuit are placed between the stimulating electrode and the return electrode, as shown in the lower right part of Fig. 3(b). Each electrode is a 85 $\times 32.5 \mu \mathrm{m}^{2}$ rectangle and the distance between the two electrodes is $20 \mu \mathrm{m}$. The distance to other electrodes in other pixels is $15 \mu \mathrm{m}$. The electrode structure with local return electrodes exhibits improved field confinement and can elicit a stronger network-mediated retinal response than those with a common remote return. ${ }^{(27)}$

\subsection{Circuit implementation}

In the proposed dual light optical system shown in Fig. 4 where the implanted chip is located in the macular region, the $850 \mathrm{~nm}$ IR light from an extraocular optical goggle is 


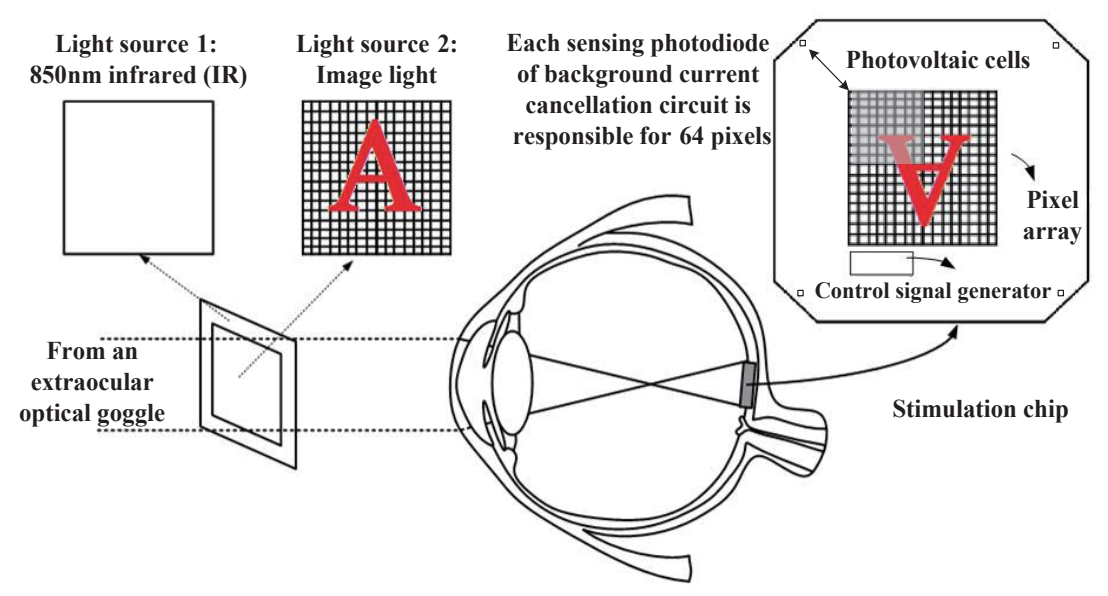

Fig. 4. (Color online) Architecture of dual light sources in the extraocular part and the implanted subretinal chip.

incident mainly on photovoltaic cells, whereas the image light is incident mainly on the pixel array. The sensing photodiode of the background current cancellation circuit is located at the corner of the chip. Each sensing photodiode is responsible for 64 pixels. The APS circuit with the background current cancellation circuit is shown in Fig. 5, where the gate node of M1 is distributed across the 64-pixel subarray. In the APS circuit, photodiode D2 is used to sense the image light and generate the photocurrent. To achieve the biphasic current stimulation, switches M10-M13 with the two nonoverlapping eight-phase clocks ( $V_{P H 1}$ and $V_{P H 2}$ for NMOS, $V_{P H 1-b a r}$ and $V_{P H 2-b a r}$ for PMOS) are used. M10 and M11 are medium-threshold-voltage PMOS devices with a threshold voltage of $-0.26 \mathrm{~V}$, whereas other PMOS devices are normal with a threshold voltage of $-0.488 \mathrm{~V}$. When $V_{P H 1}\left(V_{P H 2}\right)$ is high and $V_{P H 1-b a r}\left(V_{P H 2}-b a r\right)$ is low, M10 and M13 (M11 and M12) are on and $I_{\text {out }}$ of M16 realizes the positive (negative) stimulus on the retinal cells. When M10-M13 are off, the pixel is deselected and powered down.

The IR light is incident mainly on photovoltaic cells to generate power for the pixel circuits. However, the undesired photocurrent generated in the photodiodes of pixels by the residual or fringing IR must be cancelled. Thus, a background current cancellation circuit is added to each pixel where photodiode D1 is placed in the photovoltaic-cell array, and it has the same size as the pixel photodiode D2. The generated background current on D1 is mirrored with a suitable ratio through $\mathrm{M} 1-\mathrm{M} 2$ to the cathode of D2 to cancel $I_{b g}$ from the image photocurrent $I_{\text {Photo }}$ on D2. After the cancellation of the background current, the remaining current $I_{\text {Photo }}{ }^{-}$ $I_{b g}$ is called the signal current. This signal current is mirrored through M3-M6 and integrated on $\mathrm{C} 1$ at node A. Note that the residual intensity of IR on the pixel array should be kept small through suitable optical system design. Thus the background current cancellation can be easily realized. The background current after cancellation must be smaller than the threshold stimulation current of retinal cells.

The timing diagram showing the operation of one group of pixels is shown in Fig. 6. In the initial phase of $6.2 \mathrm{~ms}$, the stimulation currents generated by $V_{P H 1}$ and $V_{P H 2}$ are not correct. The correct stimulation begins after the reset signal $V_{P H 3-b a r}$ turns on $\mathrm{M} 7$ to reset $\mathrm{C} 1$ to $V_{D D}$ and M11 to charge the gate node B of M16 to $V_{D D}$. After the reset operation, the capacitance 


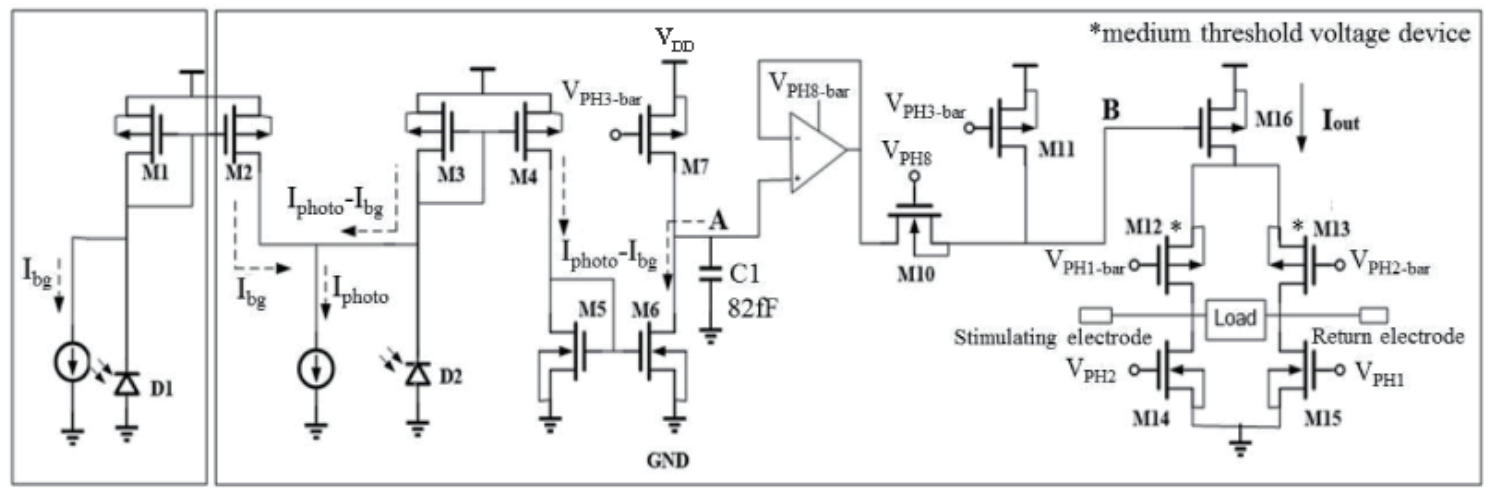

Background current cancellation circuit

Active Pixel Sensor

\begin{tabular}{lccccccc}
\hline & M1 & M2 & M3 & M4 & M5 & M6 & M7 \\
\cline { 2 - 8 } & $5 / 20$ & $5 / 20$ & $0.22 / 20$ & $0.22 / 20$ & $0.22 / 20$ & $0.22 / 20$ & $0.22 / 0.18$ \\
Dimensions & $m=2$ & $m=2$ & $m=1$ & $m=1$ & $m=1$ & $m=1$ & $m=1$ \\
\cline { 2 - 8 }$(\mu \mathrm{m})$ & M10 & M11 & M12 & M13 & M14 & M15 & M16 \\
\cline { 2 - 8 } & $0.22 / 0.18$ & $0.22 / 0.18$ & $5 / 0.25$ & $5 / 0.25$ & $10 / 0.18$ & $10 / 0.18$ & $16 / 1$ \\
& $m=1$ & $m=1$ & $m=1$ & $m=1$ & $m=1$ & $m=1$ & $m=20$ \\
\hline
\end{tabular}

Fig. 5. APS circuit with the background-current cancellation circuit.

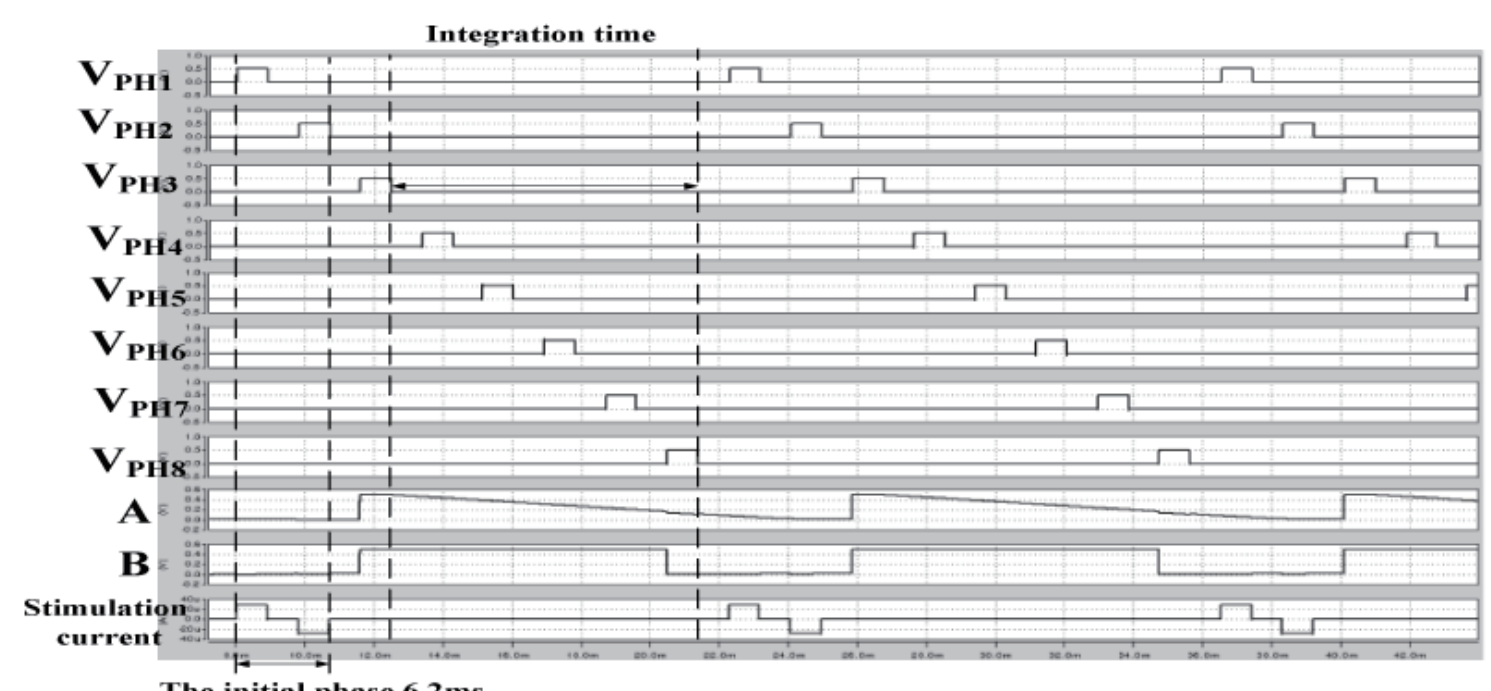

Fig. 6. Timing diagram showing the operation of one pixel group.

voltage at node $\mathrm{A}$ is discharged in accordance with the sensed photocurrent. Then the sample signal $V_{P H 8-b a r}$ turns on the op-amp unity-gain buffer to transfer the integrated voltage to node B while waiting for $V_{P H 1}$ and $V_{P H 2}$ to achieve the biphasic current stimulation. The opamp consumes $458 \mathrm{nA}$ when the sample signal $V_{P H 8-b a r}$ is low in the selected pixel. When $V_{P H 8-b a r}$ is high, the op-amp is in the power-down mode. The equivalent integration time is also indicated in Fig. 6. Compared with the design in Ref. 16, a smaller integration capacitor $\mathrm{C} 1(82 \mathrm{fF})$ is used to increase the image light sensitivity. Also, the op-amp unity-gain buffer 
is adopted to eliminate the threshold voltage drop $(\sim 0.15 \mathrm{~V})$ of the source follower in Ref. 16 to increase the dynamic range.

For the other seven groups of pixels, the operation is similar but with different clock signals. In this way, each group of pixels can function alternately, as shown in Fig. 6 and keep the same integration time.

\section{IrOx Process on Electrodes}

To deposit IrOx on electrodes of the implantable chip, the process after CMOS chip fabrication is developed. The 256-pixel implantable chip was firstly cleaned with oxygen plasma before the photolithography process. AZ5214-E was utilized as a photoresist to cover the passivation and expose the electrode array. The chip was coated with titanium (Ti) as an adhesion layer and then with IrOx under the optimized sputtering condition. ${ }^{(23)}$ The conditions of coating Ti and IrOx are shown in Table 1. After coating Ti and IrOx, AZ5214-E was removed by acetone at $30{ }^{\circ} \mathrm{C}$. Finally, the edges and bottom of the chip were sealed and protected by biocompatible silicone (NuSil Med-4840) applied by the hot-press process to prevent electrical leakage.

The same sputtering process was also performed on $1 \times 1 \mathrm{~cm}^{2}$ glass substrates to deposit $\mathrm{Ti}$ and IrOx for further material characterizations. Surface morphology and film thickness were observed under a field-emission scanning electron microscope (FE-SEM; Hitachi SU-8000). A high-resolution X-ray diffractometer (Bruker D2 Phaser) with a $1.54 \AA \mathrm{Cu} \mathrm{K} \alpha$ target was employed to identify the crystallinity of sputtering iridium oxide films (SIROFs), and the scan rate was $0.05^{\circ} \mathrm{s}^{-1}$ from 10 to 90 degrees.

Figure 7(a) shows the optical microscopic (OM) image of the 256-pixel implantable chip with an $\mathrm{IrOx} / \mathrm{Ti}$ coating on the electrode array. The OM image shows that the selective deposition process by photolithography with the AZ5214-E photoresist was successful. The IrOx/Ti layer was only deposited on the electrode area without covering the area of photodiodes or photovoltaic cells. Figure 7(b) is the cross-sectional SEM image of the IrOx/Ti layer on the glass substrate. The IrOx/Ti layer was 950/50 nm thick on the glass substrate. The depth of the trench on the 256-pixel implantable chip is about $1 \mu \mathrm{m}$ and can be filled by sputtering.

Figure 8 presents the XRD patterns of as-deposited and annealed SIROFs. The as-deposited SIROF, displayed in Fig. 8(a), shows broader characteristic diffraction peaks of IrOx, which means the crystallinity of the as-deposited SIROF is inadequate and some defects exist in the film. The crystallinity of the SIROF is greatly improved by the annealing treatment at $450{ }^{\circ} \mathrm{C}$

Table 1

Sputtering conditions of $\mathrm{Ti}$ and IrOx coatings.

\begin{tabular}{lcc}
\hline Parameter & $\mathrm{Ti}$ & $\mathrm{IrOx}$ \\
\hline Gas & $\mathrm{Ar}$ & $\mathrm{O}_{2}$ \\
Gas flow rate (sscm) & 20 & 10 \\
Pressure (Pa) & 0.6 & 1.0 \\
Power (W) & $\mathrm{DC} 100$ & $\mathrm{RF} 200$ \\
Time (min) & 5 & 60 \\
Thickness (nm) & 50 & 950 \\
\hline
\end{tabular}




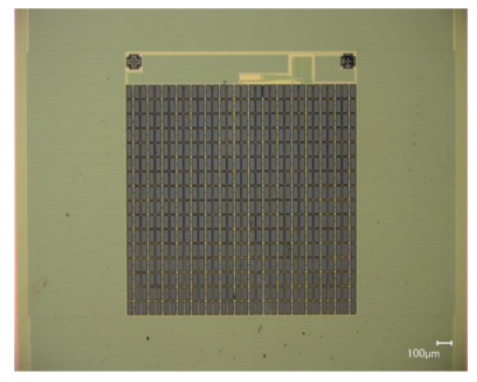

(a)

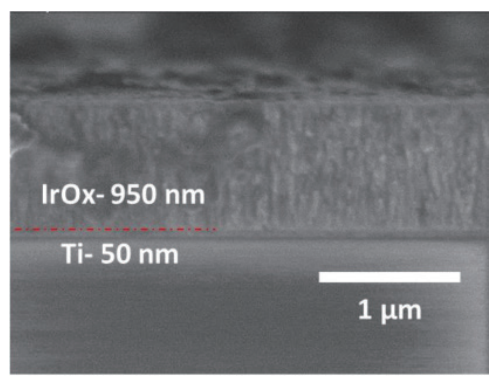

(b)

Fig. 7. (Color online) (a) OM and (b) SEM images of the 256-pixel implantable chip after applying IrOx coating.

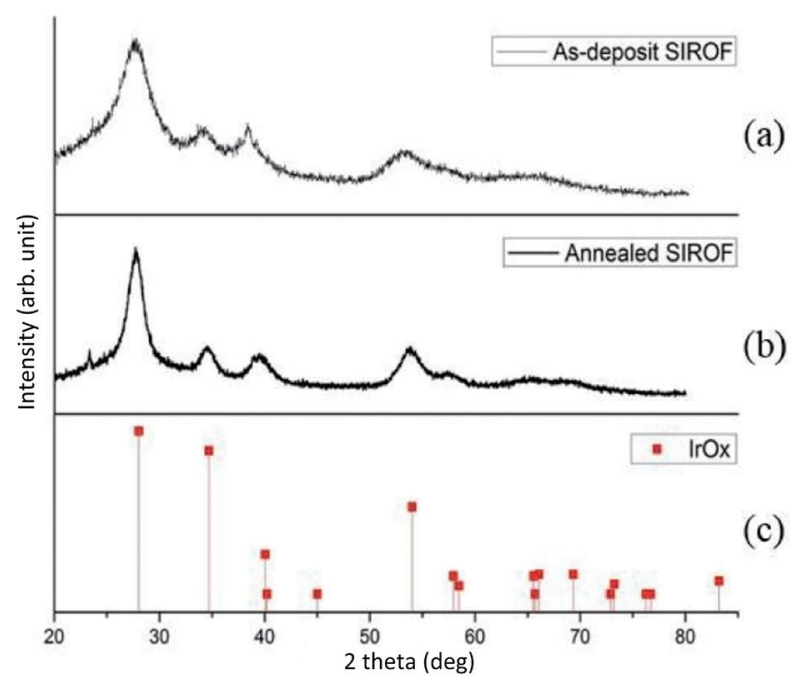

Fig. 8. (Color online) XRD patterns of (a) as-deposited SIROF and (b) annealed SIROF as well as (c) standard $\mathrm{IrO}_{2}$ patterns from JCPDS 00-043-1019.

for $2 \mathrm{~h}$ in air. Figure 8(b) reveals that the SIROF was crystallized by annealing and the resulting SIROF appears to have the rutile structure (rutile $\mathrm{IrO}_{2}$, JCPDS 00-043-1019). Therefore, the crystallized SIROF is expected to have fewer structural defects and improved properties.

Cyclic voltammetry (CV) was carried out in a $0.1 \mathrm{M}$ phosphate-buffered saline (PBS) electrolyte with a potentiostat. The working electrodes were $1 \times 1 \mathrm{~cm}^{2}$ of the as-deposited and annealed SIROFs with a thickness of $1 \mu \mathrm{m}$. A platinum sheet was used as a counterelectrode and an $\mathrm{Ag} / \mathrm{AgCl}$ electrode as a reference electrode. The charge storage capacity (CSC) was determined by the integration of cathodic current in a potential window for iridium oxide between $-0.6-0.8 \mathrm{~V}$ at $50 \mathrm{mVs}^{-1}$. It has become a common practice to characterize the stimulation electrodes by measuring the cathodic CSC. The CSC can be calculated by

$$
C S C=\frac{1}{v A} \int_{E_{c}}^{E_{a}}|i| d E,
$$


where $E$ is the electrode potential, $i$ is the measured current (A), $E_{a}$ and $E_{c}$ are the anodic and cathodic potential limits (V), respectively, $A$ is the surface area of the SIROF electrode $\left(\mathrm{cm}^{2}\right)$, and $v$ is the scan rate. The CSC value is essentially a relative measurement of the total amount of charge available for a stimulation pulse. It can be considered as a prediction of the charge injection capability for neural stimulation. An ideal stimulating electrode is expected to have a large CSC value. The operating potential must avoid the water electrolysis window, which is $-0.6-0.8 \mathrm{~V}$ vs $\mathrm{Ag} / \mathrm{AgCl}$, for the as-deposited and annealed SIROFs. The use of potential higher than this window could be accompanied by significant $\mathrm{pH}$ changes and hydrogen or oxygen evolution that could be harmful to the tissue and the implanted electrode integrity.

Figure 9 shows the measured cyclic voltammograms of the as-deposited SIROF (black dashed line) and annealed SIROF (red dashed line). Within the water electrolysis window, the CSC values obtained at $50 \mathrm{mV} / \mathrm{s}$ are 50.28 and $17.28 \mathrm{mC} / \mathrm{cm}^{2}$ for as-deposited and annealed 1 $\mu \mathrm{m}$ SIROFs, respectively. These values imply that both SIROFs potentially have good charge injection performance compared with Pt electrodes that only provide $1.99 \mathrm{mC} / \mathrm{cm}^{2}$ of CSC. ${ }^{(28)}$ The range of CSC values of $\mathrm{IrOx}$ is very wide, from 2.8 to $150 \mathrm{mC} / \mathrm{cm}^{2}$. In the literature, the deposition conditions of IrOx sputtering strongly influence the resulting CSC. ${ }^{(21,24)}$ In addition, the CSC value of the as-deposited SIROF was larger than that of the annealed SIROF because the structure became more crystalline, thereby reducing both the faradaic reaction and the CSC value. In fact, we carried out the annealing for materials characterization and the temperature was too high for the DPSS chip. As a result, the CSC value of the annealed IrOx electrode became smaller. Therefore, annealing will not be necessary or applied in the future development.

The biocompatibility was evaluated through the MTT [3-(4,5-dimethylthiazol-2-yl)-2,5diphenyltetrazolium bromide; $\left.\mathrm{C}_{18} \mathrm{H}_{16} \mathrm{BrN}_{5} \mathrm{~S}\right]$ cell viability test. Both as-deposited and annealed SIROFs over an area of $1 \times 1 \mathrm{~cm}^{2}$ were prepared for the cell viability test and glass was used

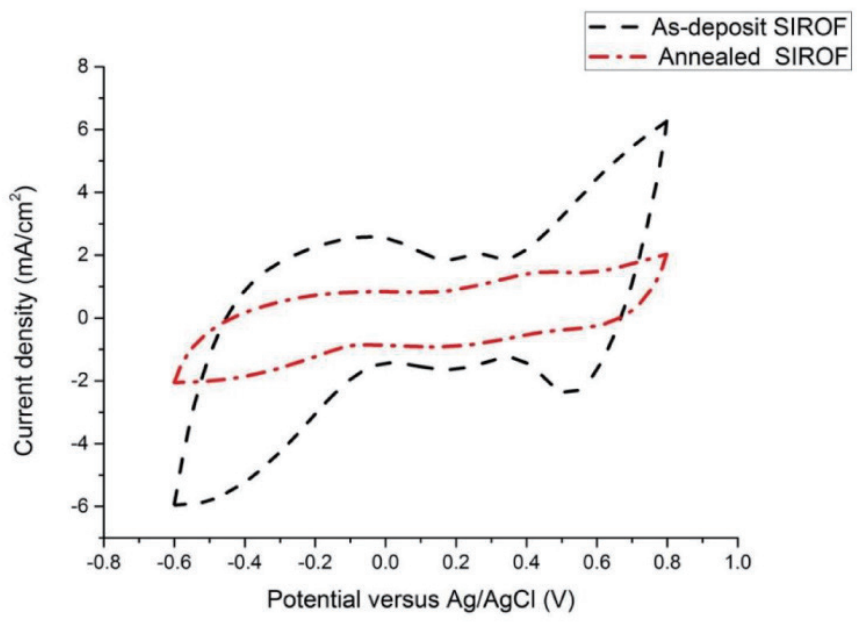

Fig. 9. (Color online) Measured cyclic voltammograms of as-deposited SIROF (black dashed line) and annealed SIROF (red dashed line). 
as the control group. The adrenal pheochromocytoma (PC-12) cells were seeded on the SIROF samples and glass in 24-well plates at a density of 50000 cells per well in the presence of Dulbecco's modified Eagle's medium (DMEM, $0.5 \mathrm{~mL}$ ) containing 10\% fetal bovine serum (FBS) and 1\% penicillin/streptomycin. The reason why PC12 was used for the MTT test is that PC12 is a neuron-like cell and the application of DPSS chips is for neurostimulation. After incubation for $24 \mathrm{~h}$, the medium was replaced with fresh medium supplemented with MTT (4 $\mathrm{mg} \mathrm{mL}^{-1}$, ${ }^{(28-30)} 0.5 \mathrm{~mL}$ per well). After another $4 \mathrm{~h}$, the medium containing MTT was removed and dimethyl sulfoxide (DMSO) $(0.5 \mathrm{~mL}$ per well) was added to dissolve the formazan crystals. Each of the 24 wells was transferred to a 96 -well plate. The optical density of the resulting solution was measured with a $595 \mathrm{~nm}$ light source using an absorbance microplate reader (Infinite F50, TECAN). ${ }^{(28-30)}$ The cell viability percentage was calculated by the following formula:

$$
\text { Cell viability percentage }(\%)=\frac{\text { ODsample }}{\text { ODcontrol }} \times 100 \% \text {. }
$$

Figure 10 shows the results of cell viability for as-deposited SIROF and is over $100 \%$ although there was no statistical significance. It seems that the SIROF provides better biocompatibility than a glass substrate. Thus, it is suggested that the SIROF demonstrates good biocompatibility and potential compatibility for the growth and differentiation of neurons.

\section{Electrical Test and In Vivo Test}

The measurement setup for electrical testing of the fabricated 256-pixel subretinal chip is shown in Fig. 11(a) where an $850 \mathrm{~nm}$ IR LED is installed inside the optical instrument to provide the background IR for the on-chip photovoltaic cells. The image light is the visible red light from a $643 \mathrm{~nm}$ red LED light source and is incident on the pixel array through a convex focal lens to ensure the location of the spot. The pixel array converts the image light into photocurrents for electrical stimulation. As shown in Fig. 11(a), the red LED light is reflected by two mirrors to project mainly on the pixel array of the chip shown in Fig. 11(b). The IR LED light is reflected by a hot mirror and is projected on the whole chip as the worst-case

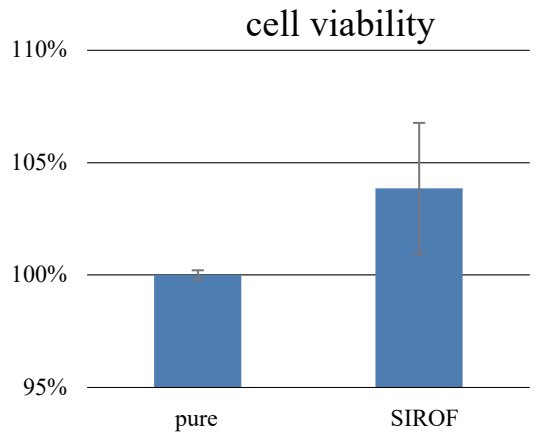

Fig. 10. (Color online) Cell viability of SIROF. 


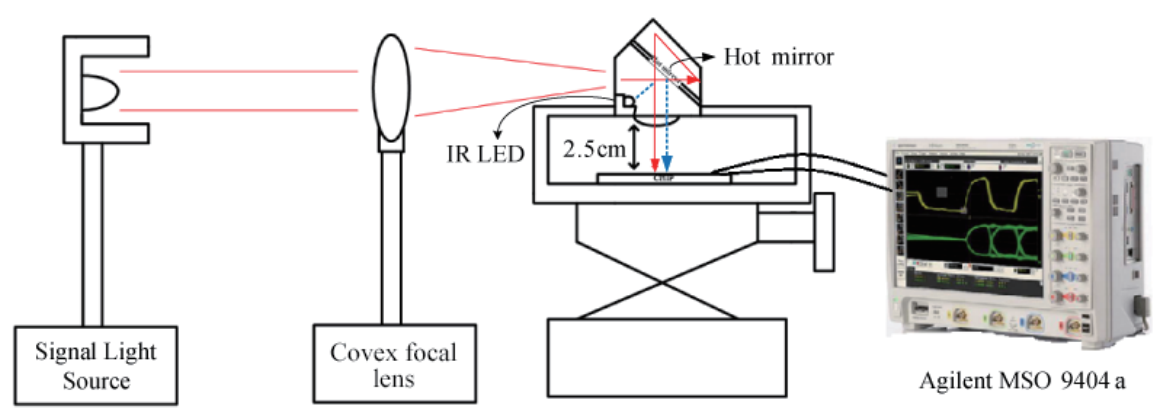

(a)

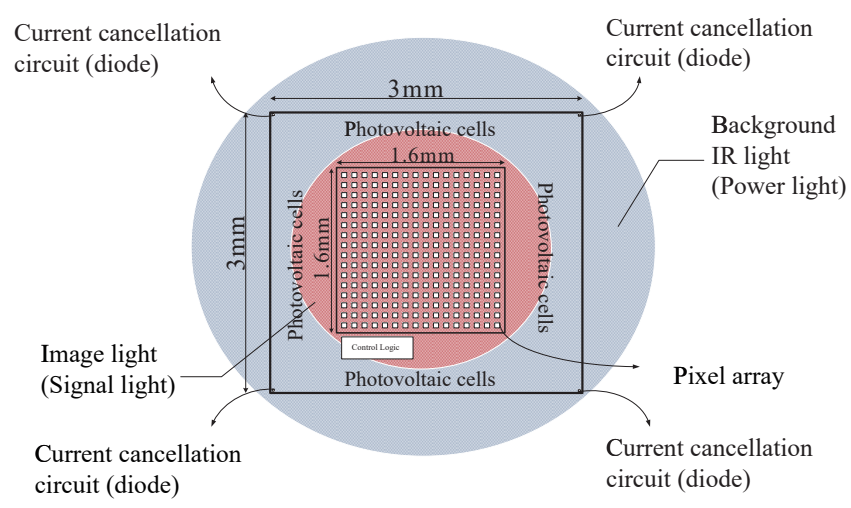

(b)

Fig. 11. (Color online) (a) Measurement setup for electrical test with dual light source system. (b) Subretinal chip in dual light source system.

measurement with the strongest background IR intensity. In the measurement of electrical signals, the signal light intensity is $0.4 \mathrm{~mW} / \mathrm{cm}^{2}$ and the intensity of the background IR is 80 $\mathrm{mW} / \mathrm{cm}^{2}$.

The photocurrent generated from on-chip photovoltaic cells is measured from a test-key chip with 138600 photovoltaic cells connected in parallel. In the measurement setup, an Agilent B1505A power device analyzer is used to measure the $I-V$ curve of photovoltaic cells when the IR LED illuminates the chip through the optical instrument. The total generated current of photovoltaic cells versus IR intensity is shown in Fig. 12. The background IR intensity of $80 \mathrm{~mW} / \mathrm{cm}^{2}$ was chosen to provide the supply current of $1.4 \mathrm{~mA}$. Under the background IR intensity of $80 \mathrm{~mW} / \mathrm{cm}^{2}$, the maximum current of the sensing photodiode is $576 \mathrm{pA}$. We choose the signal light intensity of $0.4 \mathrm{~mW} / \mathrm{cm}^{2}$ to provide the signal current of $6.4 \mathrm{pA}$, which is sufficient to yield the maximum stimulation current.

The electrical test is performed to measure the stimulation current of a single pixel for functional verification. The electrode impedance model is shown in Fig. 13. The measured biphasic stimulation currents are shown in Fig. 14(a) for the case where a load resistor of $10 \mathrm{k} \Omega$ was connected between the two electrodes. The measured voltage waveform is $199.5 \mathrm{mV}$, which is equivalent to the stimulation current of $19.95 \mu \mathrm{A}$. The stimulation frequency is $30.2 \mathrm{~Hz}$ and the amount of injected charges at each pixel is $41.28 \mathrm{nC}$. The pixel-array power consumption 

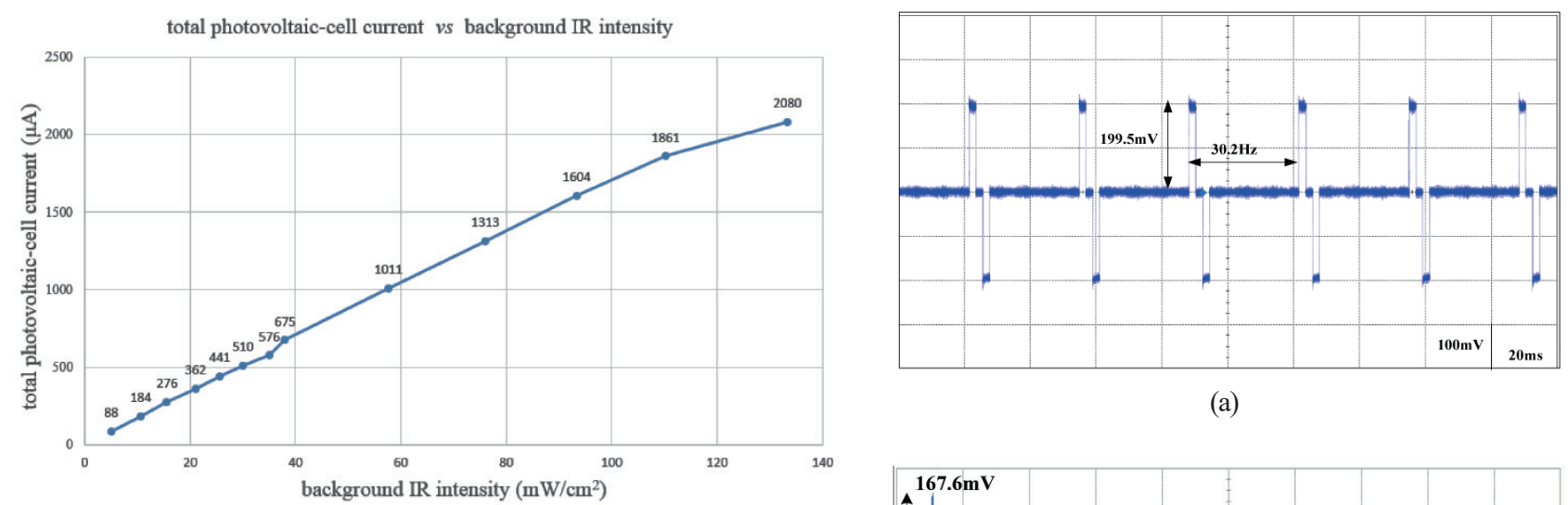

(a)

Fig. 12. (Color online) Measured total photovoltaiccell current under different background IR intensities.

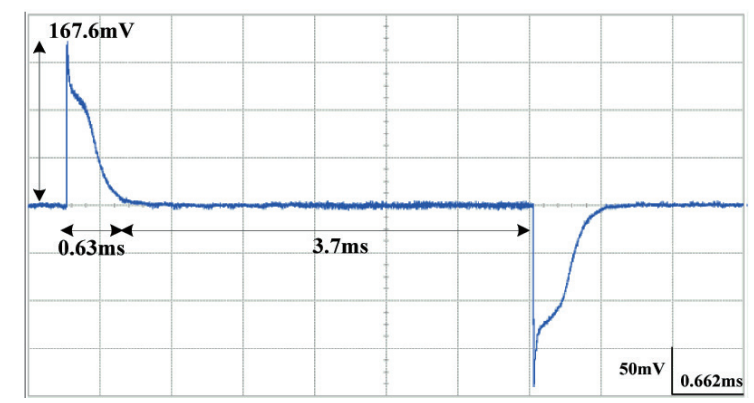

(b)
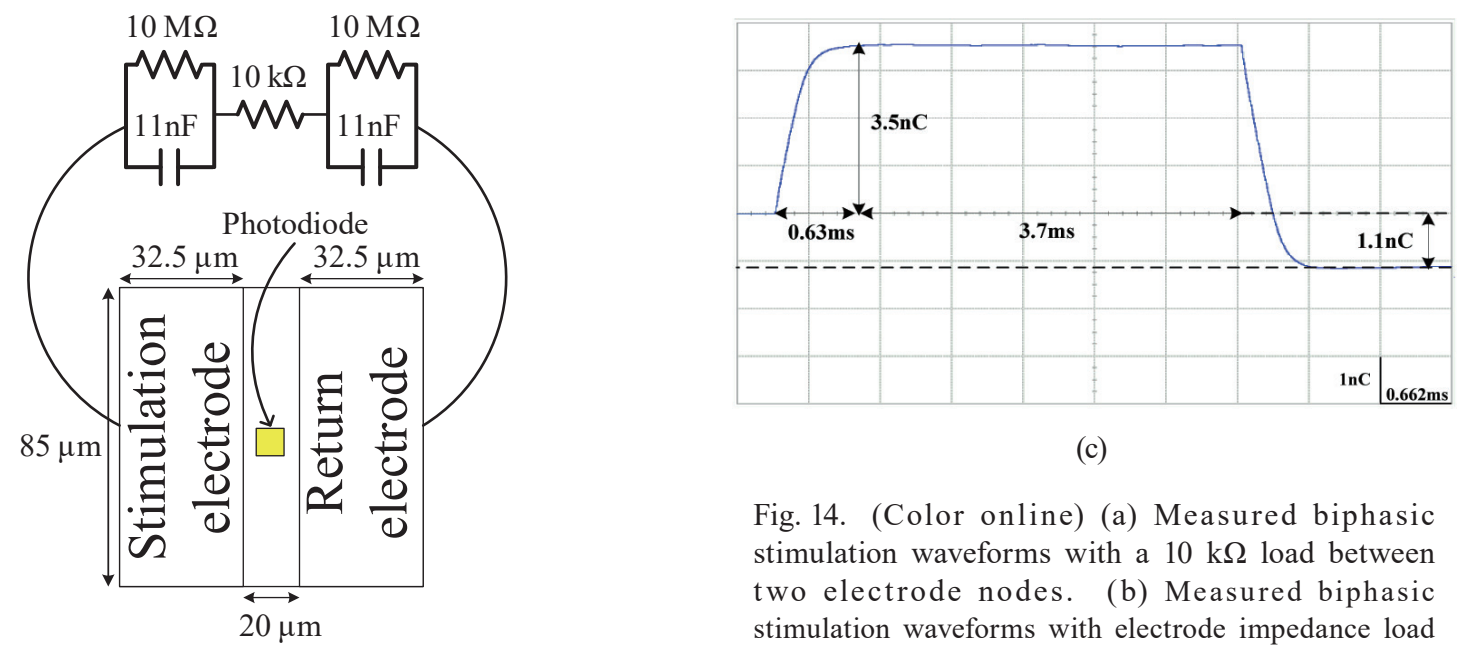

(c)

Fig. 14. (Color online) (a) Measured biphasic stimulation waveforms with a $10 \mathrm{k} \Omega$ load between two electrode nodes. (b) Measured biphasic stimulation waveforms with electrode impedance load between two electrode nodes. (c) Measured injected charge under electrode impedance load.

Fig. 13. (Color online) Electrode impedance model.

is $638 \mu \mathrm{W}$. With the equivalent electrode impedance connected between two electrode nodes, the measured biphasic stimulation waveforms are shown in Fig. 14(b). As seen in Fig. 14(b), the measured peak voltage waveforms is $167.6 \mathrm{mV}$, which is equivalent to the stimulation current of $16.76 \mu \mathrm{A}$. The injected charge at each pixel is $3.5 \mathrm{nC}$, as shown in Fig. 14(c). In the measurement result, the charge imbalance is $1.1 \mathrm{nC}$ in $34.64 \mathrm{~ms}$, which is equal to $31.7 \mathrm{nC} / \mathrm{s}$. Compared with that in Ref. 31, the measured charge imbalance is much smaller than $10 \mu \mathrm{C} / \mathrm{s}$. Compared with the other subretinal implant structure without external wired power, ${ }^{(12)}$ the proposed chip structure realizes biphasic stimulation and has a better image sensitivity because 
of the use of the APS technique. Compared with that in Ref. 16, the image sensitivity is 12.5 times higher.

The injected charges through electrodes are $3.5 \mathrm{nC} /$ pixel, which is above the threshold charge of approximately $1 \mathrm{nC}$ per electrode. ${ }^{(32)}$ Since the threshold charges for successful stimulation differ for each patient, the increase in injected charge is necessary. This can be achieved by either increasing the electrode area by using bump electrodes ${ }^{(22-24)}$ or increasing the stimulation voltage by using charge pump circuits. These improvements will be realized in the future.

In the in vivo animal test, one Lanyu minipig was implanted with the retinal chip. The electrodes of the chip were coated with IrOx by RF sputtering. All the procedures were in accordance with the Association for Research in Vision and Ophthalmology's statement for the use of animals in ophthalmic and vision research, and were approved by the Laboratory Animal Care and Use Committee of Taipei Veterans General Hospital (IACUC number: 2015-047) as well as by the Laboratory Animal Care and Use Committee of Pigmodel Animal Technology (AAALAC number: 001636, IACUC number: PIG-104006 \& PIG-106008).

The pig was 1.5 years old with a body weight $65 \mathrm{~kg}$. Before the experiment, the pig was tranquilized by intramuscular injection of Azeperonum 3-5 and $0.03-0.05 \mathrm{mg} / \mathrm{kg}$ atropine. Then anesthesia was carried out by intramuscular injection of Schotta-50 (Zoletil@-50), at a dose of 3-5 mg/kg. Subsequently, the pig trachea was intubated and inflated with oxygen/ nitrous oxide gas (2:1), which was further mixed with $0.5-2 \%$ isoflurane, at a rate of $2-3 \mathrm{~L} / \mathrm{min}$.

The implantation procedures start with making a scleral opening and a choroidal cut over the eyeball equator externally, and creating an artificial retinal detachment with saline. Then the biocompatible chip is inserted through the sclera-choroid opening and placed in the posterior pole of the eyeball, as shown in Fig. 15. After surgery, sub-Tennon injection of gentamicin and dexamethasone is performed. The pig was individually housed for postoperative care and given aspirin or acetaminophen analgesics.

Two months and seven months after the implantation, electroretinography (ERG) was carried out to verify the function of the chip. The ERG apparatus was RETI-port/scan21 (Roland Consult, Germany) with Ganzfeld Q450SC. The electrode for ERG recording was the BurianAllen (Hansen Ophthalmic Development Lab., USA) type, and the ERG system was grounded

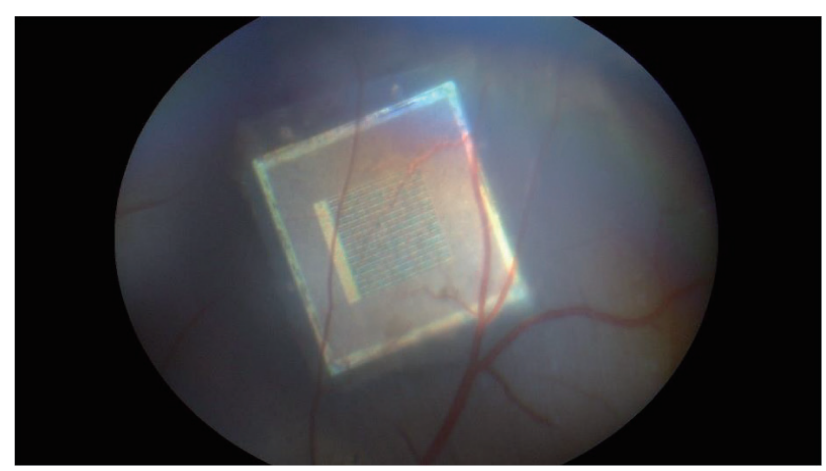

Fig. 15. (Color online) One week after implantation of the 256-pixel implantable chip. Retina is well attached and each pixel of the chip is clearly seen. 
with a needle electrode placed subcutaneously in the back.

The intensity of the standard flash is $3.0 \mathrm{~cd} / \mathrm{m}^{2}$ (0 dB, ISCEV standard flash). ERG was carried out after bleaching with $450 \mathrm{~cd} / \mathrm{m}^{2}$ white light for one hour. The ERG measurement conditions were continuous $450 \mathrm{~cd} / \mathrm{m}^{2}$ white light as the background and $-20 \mathrm{~dB}$ flash white light as the stimulation. Ten serial flashes at intervals of $3 \mathrm{~s}$ were applied and the waves were amplified and averaged. The pig was kept for 7 months.

ERG could be triggered and recorded from 2 to 7 months after implantation. There was no significant eye inflammation or adverse event throughout the whole follow-up term of 7 months. ERG could be recorded with a very low intensity of light triggering, such as $-20 \mathrm{~dB}$, after light bleaching. In contrast, it could not be recorded in the contralateral eye (the control). There was no significant chip dislocation.

The experimental eye showed a spike response similar to the b-wave, whereas the control eye showed a flat response. Under the ERG recording condition with continuous $450 \mathrm{~cd} / \mathrm{m}^{2}$ white light as the background and with $-20 \mathrm{~dB}$ flash white light stimulation, two months after chip implantation, the implicit time of the a-wave was $22.3 \mathrm{~ms}$, and the b-wave implicit time was $26.1 \mathrm{~ms}$ with an amplitude of $0.77 \mu \mathrm{V}$, as shown in Fig. 16. Seven months after implantation, with $-15 \mathrm{~dB}$ stimulation, the implicit time of the a-wave was $15.3 \mathrm{~ms}$, and the b-wave implicit time was $22.6 \mathrm{~ms}$ with an amplitude of $0.95 \mu \mathrm{V}$, as shown in Fig. 17. Seven months after implantation, with $-20 \mathrm{~dB}$ stimulation, the implicit time of the a-wave was 13.2 $\mathrm{ms}$, and the b-wave implicit time was $22.3 \mathrm{~ms}$ with an amplitude of $0.82 \mu \mathrm{V}$, as shown in Fig. 18 . In the control eye with $0 \mathrm{~dB}$ stimulation, the implicit time of the a-wave was generally around $14 \mathrm{~ms}$, and the implicit time of the b-wave was generally around $25 \mathrm{~ms}$. It is reasonable that the b-wave amplitudes are small, since the retina area overriding the chip was only a small portion of the whole retina. The $\mathrm{S} / \mathrm{N}$ in the test was about $5.25 \mathrm{for}-15 \mathrm{~dB}$ stimulation and 4.75 for -20 $\mathrm{dB}$ stimulation.
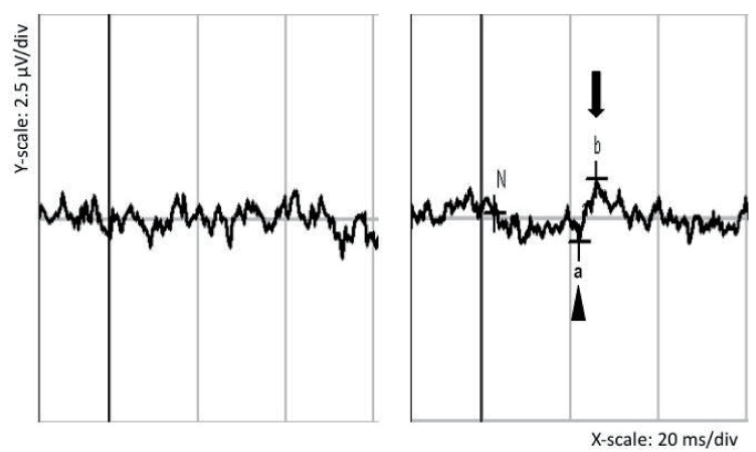

Fig. 16. Right image shows the measured ERG two months after implantation of the 256-pixel implantable chip with $-20 \mathrm{~dB}$ light stimulation. Left image shows the measured ERG of the contralateral eye as the control with the same stimulation. The implicit time of the a-wave is $22.3 \mathrm{~ms}$. The implicit time of the b-wave is $26.1 \mathrm{~ms}$ and the amplitude is $0.77 \mu \mathrm{V}$. Arrowhead: a-wave. Arrow: b-wave.
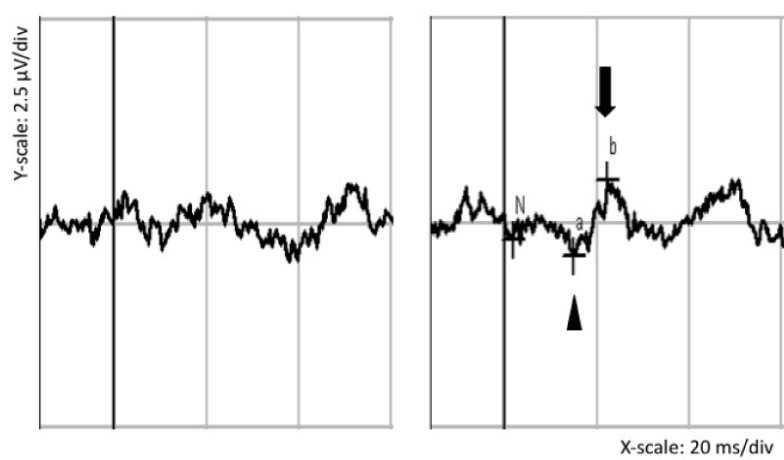

Fig. 17. Right image shows the measured ERG seven months after implantation of the 256-pixel implantable chip with $-15 \mathrm{~dB}$ light stimulation. Left image shows the measured ERG of the contralateral eye as the control with the same stimulation. The implicit time of the a-wave is $15.3 \mathrm{~ms}$. The implicit time of the b-wave is $22.6 \mathrm{~ms}$ and the amplitude is $0.96 \mu \mathrm{V}$. Arrowhead: a-wave. Arrow: b-wave. 

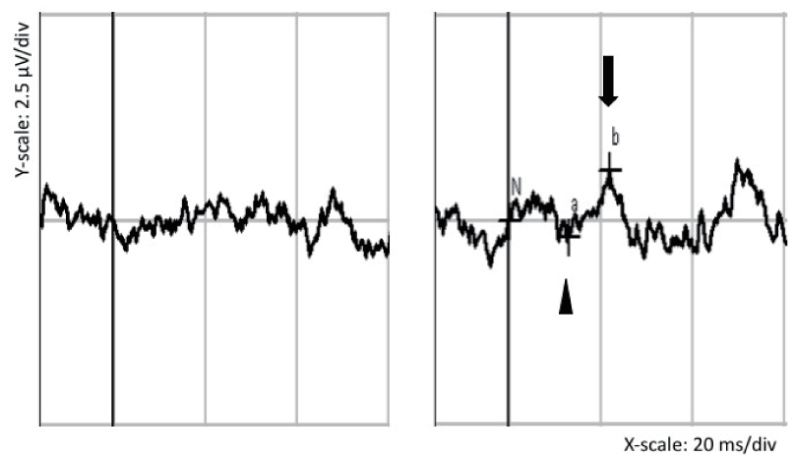

Fig. 18. Right image shows the measured ERG seven months after implantation of the 256-pixel implantable chip with $-20 \mathrm{~dB}$ light stimulation. Left image shows the measured ERG of the contralateral eye as the control with the same stimulation. The implicit time of the a-wave is $13.2 \mathrm{~ms}$. The implicit time of the b-wave is $22.3 \mathrm{~ms}$ and the amplitude is $0.96 \mu \mathrm{V}$. Arrowhead: a-wave. Arrow: b-wave.

In this system, only insignificant a-waves were recorded, because the whole retina was bleached and the triggering light intensity is too weak to induce any a-waves from the retina. After two or seven months' implantation of the subretinal chip, there might be only a few photoreceptors remaining in the overriding retina. The recorded $b$-wave was triggered from the implanted retinal chip, which could not produce any a-wave.

\section{Conclusions}

A CMOS self-powered 256-pixel implantable chip with on-chip photovoltaic cells, electrodes with IrOx coating, and APSs was proposed for subretinal prostheses. From the electrical measurement results, it was shown that under a $10 \mathrm{k} \Omega$ load, the fabricated chip had an output stimulation current of $19.95 \mu \mathrm{A}$. Under the equivalent electrode impedance load, the fabricated chip had a peak output stimulation current of $16.7 \mu \mathrm{A}$. The stimulation frequency was $30.2 \mathrm{~Hz}$ and the amount of injected charges at each pixel was $3.5 \mathrm{nC}$. Both image light sensitivity and injection charges were significantly improved. A successful fabrication process for IrOx film on electrodes via sputtering deposition was developed. The surface morphology, crystallinity, CSC, and biocompatibility of SIROFs were investigated. As a result, the SIROF was found to have desirable physical and electrochemical properties making it a unique candidate for the neurostimulation electrodes in the 256-pixel implantable chip. The chip demonstrated 7-month biocompatibility and charge delivery capability, which were also confirmed by the measured ERG of a Lanyu minipig in the in vivo animal tests.

\section{Acknowledgments}

The authors thank the Chip Implementation Center (CIC), Taiwan, for the fabrication of the chip. This work was supported in part by the Novel Bioengineering and Technological Approaches to Solve Two Major Health Problems in Taiwan sponsored by the Taiwan Ministry of Science and Technology Academic Excellence Program under Grant Number: MOST 106- 
2633-B-009-001. This work was supported in part by the National Research Program for Biopharmaceuticals under Grant Number: MOST 104-2325-B-010-005-.

\section{References}

1 K. Chen, Z. Yang, L. Hoang, J. Weiland, M. Humayun, and W. Liu: Proc. 2013 IEEE Int. Solid-State Circuits Conf. (IEEE, 2013) 294-296.

2 W. Liu, K. Vichienchom, M. Clements, S. C. DeMarco, C. Hughes, E. McGucken, M. S. Humayun, E. De Juan, J. D.Weiland, and R. Greenberg: IEEE J. Solid-State Circuits 35 (2000) 1487.

3 K. Chen, Z. Yang, L. Hoang, J. Weiland, M. Humayun, and W. Liu: IEEE J. Solid-State Circuits 45 (2010) 1946.

4 M. Ortmanns, A. Rocke, M. Gehrke, and H. J. Tiedtke: IEEE J. Solid-State Circuits 42 (2007) 2946.

5 T. Lehmann, N. H. Lovell, G. J. Suaning, P. Preston, Y. T. Wong, N. Dommel, L. H. Jung, Y. Moghe, and K. Das: Proc. 2008 IEEE Int. Symp. Circuits and Systems (IEEE, 2008) 352-355.

6 W. Mokwa, M. Goertz, C. Koch, I. Krisch, H. K. Trieu, and P. Walter: Proc. IEEE Engineering in Medicine and Biology Society (IEEE, 2008) 5790-5793.

7 L. S. Theogarajan: IEEE J. Solid-State Circuits 43 (2008) 2322.

8 C. C. Chiao, Y. T. Yang, C. Wan, W. C. Yang, L. J. Lin, P. K. Lin, and C. Y. Wu: Invest. Ophthalmol. Visual Sci. 51 (2010) 3021.

9 K. Mathieson, J. Loudin, G. Goetz, P. Huie, L. Wang, T. I. Kamins, L. Galambos, R. Smith, J. S. Harris, A. Sher, and D. Palanker: Nat. Photonics 6 (2012) 391.

10 C. L. Lee and C. C. Hsieh: IEEE Trans. Electron Devices 60 (2013) 1162.

11 P. K. Lin, P. H. Kuo, Y. C. Tsai, M. J. Sui, C. C. Chiao, T. Noda, J. Ohta, and C. Y. Wu: Proc. 2014 World Research Congress: The Eye and The Chip (2014).

12 C. Y. Wu, P. H. Kuo, P. K. Lin, Y. C. Huang, C. K. Su, H. Li, C. C. Chiao, Y. T. Huang, Y. C. Tsai, J. W. Pan, T. Noda, and J. Ohta: Proc. 2014 World Research Congress: The Eye and The Chip (2014).

13 S. Oh, J. H. Ahn, S. Lee, H. Ko, J. M. Seo, Y. S. Goo, and D. Cho: IEEE Trans. Biomed. Eng. 62 (2015) 70.

14 E. Zrenner, K. U. Bartz-Schmidt, H. Benav, D. Besch, A. Bruckmann, V. P. Gabel, F. Gekeler, U. Greppmaier, A. Harscher, S. Kibbel, J. Koch, A. Kusnyerik, T. Peters, K. Stingl, H. Sachs, A. Stett, P. Szurman, B. Wilhelm, and R. Wilke: Proc. R. Soc. London, Ser. B 278 (2011) 1489.

15 K. Stingl, K. U. Bartz-Schmidt, D. Besch, A. Braun, A. Bruckmann, F. Gekeler, U. Greppmaier, S. Hipp, G. Hörtdörfer, C. Kernstock, A. Koitschev, A. Kusnyerik, H. Sachs, A. Schatz, K. T. Stingl, T. Peters, B. Wilhelm, and E. Zrenner: Proc. R. Soc. London, Ser. B 280 (2013) 20130077.

16 C. Y. Wu, W. J. Sung, P. H. Kuo, C. K. Tzeng, C. C. Chiao, and Y. C. Tsai: Proc. 2015 IEEE Biomedical Circuits and Systems Conf. (IEEE, 2015) 1-4.

17 J. Pine: J. Neurosci. Methods 2 (1980) 19.

18 C. A. Thomas Jr., P. A. Springer, G. E. Loeb, Y. Berwald-Netter, and L. M. Okun: Exp. Cell. Res. 74 (1972) 61.

19 M. P. Maher, H. Dvorak-Carbone, J. Pine, J. A. Wright, and Y. C. Tai: Med. Biol. Eng. Comput. 37 (1999) 110.

20 D. R. Merrill, M. Bikson, and J. G. R. Jefferys: J. Neurosci. Methods 141 (2005) 171.

21 S. F. Cogan: Annu. Rev. Biomed. Eng. 10 (2008) 275.

22 Y. L. Pan, T. Noda, K. Sasagawa, T. Tokuda, H. Kanda, T. Fujikado, and J. Ohta: J. Phys. Conf. Ser. 352 (2012) 012005.

23 Y. L. Pan, T. Noda, K. Sasagawa, T. Tokuda, and J. Ohta: IEEJ Trans. Electr. Electr. Eng. 8 (2013) 310.

24 T. Noda, K. Sasagawa, T. Tokuda, Y. Terasawa, H. Tashiro, H. Kanda, T. Fujikado, and J. Ohta: Sens. Actuators, A 211 (2014) 27.

25 J. D. Weiland, D. J. Anderson, and M. S. Humayun: IEEE Trans. Biomed. Eng. 49 (2002) 1574.

26 M. Coltheart: Philos. Trans. R. Soc. London, Ser. B 290 (1980) 57.

27 F. Thomas, G. Georges, L. Xin, and P. Daniel: J. Neural Eng. 13 (2016) 036010.

28 Y. M. Chen, T. W. Chung, P. W. Wu, and P. C. Chen: J. Alloys Compd. 692 (2017) 339.

29 X. Li, Y. Kuang, H. C. Lin, Y. Gao, J. Shi, and B. Xu: Angew. Chem. Int. Ed. Engl. 50 (2011) 9365.

30 Y. H. Liu, S. M. Hsu, F. Y. Wu, H. Cheng, M. Y. Yeh, and H. C. Lin: Bioconjug Chem. 25 (2014) 1794.

31 Neuropace Company: http://www.neuropace.com/wp-content/uploads/2015/11/UserManual.pdf (accessed April 2015).

32 F. Gekeler, K. Kobuch, H. N. Schwahn, A. Stett, K. Shinoda, and E. Zrenner: Graef. Arch. Clin. Exp. Ophthalmol. 242 (2004) 587. 


\section{About the Authors}

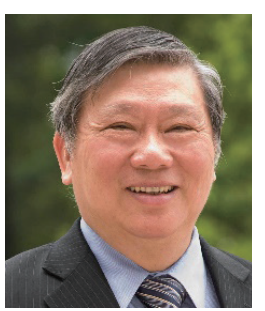

Chung-Yu Wu received his M.S. and Ph.D. degrees from the Department of Electronics Engineering, National Chiao Tung University, Hsinchu, Taiwan, R.O.C., in 1976 and 1980, respectively. Since 1980, he has served as a consultant to high-tech industry and research organizations and has built up strong research collaborations with high-tech industries. From 1980 to 1983, he was an associate professor at National Chiao Tung University. During 1984 to 1986, he was a visiting associate professor in the Department of Electrical Engineering, Portland State University, Portland, OR. Since 1987, he has been a professor at National Chiao Tung University. From 1991 to 1995, he was rotated to serve as the Director of the Division of Engineering and Applied Science on the National Science Council, Taiwan. From 1996 to 1998, he was honored as the Centennial Honorary Chair Professor at National Chiao Tung University. He received the National Chair Professorship from the Ministry of Education, 2015-2017. Currently, he is a Life Chair Professor at National Chiao Tung University. He has published more than 300 technical papers in international transactions/ journals and conferences. He also has 47 patents including 23 U.S. patents. His research interests are implantable biomedical integrated circuits and systems, intelligent bio-inspired sensor systems, RF/microwave communication integrated circuits, neural network, analog/ mixed-signal integrated circuits, and nanoelectronics. He is a member of Eta Kappa Nu and Phi Tau Phi Honorary Scholastic Societies. He was a recipient of the IEEE Fellow Award in 1998 and Third Millennium Medal in 2000. In Taiwan, he has received numerous research awards from the Ministry of Education, National Science Council, and professional foundations.

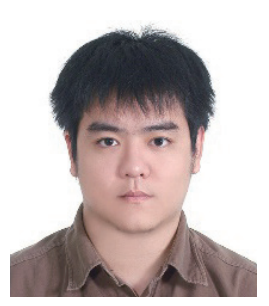

Po-Han Kuo received his B.S. degree in electronics engineering from National Changhua University of Education, Changhua, Taiwan, in 2009 and M.S. degree from the Insititute of Biomedical Engineering of National Yang Ming University, Hsinchu, Taiwan, in 2011. He is now working towards his Ph.D. degree at the Institute of Electronics, National Chiao Tung University. His major research interests are retinal prosthesis and biomedical electronics.

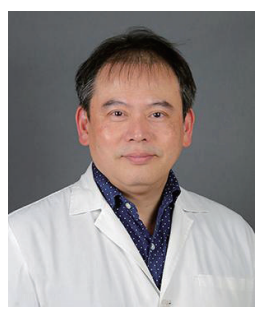

Po-Kang Lin received his M.D. degree from National Yang-Ming University, Taiwan, in 1985. He has been a resident, the chief resident, and a retinal fellow successively at the Department of Ophthalmology, Taipei Veterans General Hospital, Taiwan, since 1987. Subsequently, he has been an attending doctor in the retina section since 1993. Thereafter, he became an associate professor at National Yang-Ming University, and a professor at Defense University, Taiwan. His research interests are in clinical ophthalmology, retinal biology, and biomedical engineering. 


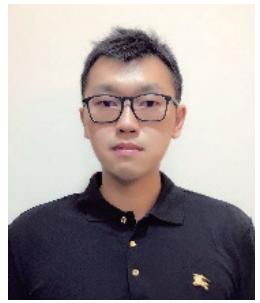

Po-Chun Chen received his B.S. and M.S. degrees from National Chiao Tung University, Taiwan, in 2006 and 2008, respectively. He obtained his Ph.D. degree from Texas A\&M University, USA, in 2014. From 2014 to 2016, he was an assistant research fellow at National Chiao Tung University, Taiwan. Since 2016, he has been an assistant professor at National Taipei University of Technology, Taiwan. His research interests are in biomaterials for implantable medical devices.

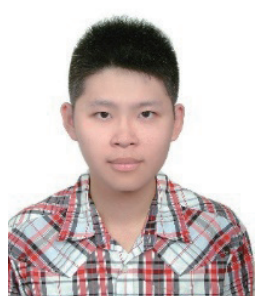

Wei-Jie Sung received his B.S. degree from National Chung Hsing University, Taiwan, in 2013 and his M.S. degree from the National Chaio Tung University, Taiwan, in 2016. His research interests are in bioengineering and sensors.

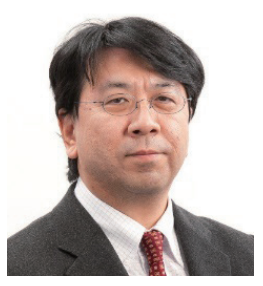

Jun Ohta received his B.E., M.E., and Dr. Eng. degrees in applied physics, all from the University of Tokyo, Japan, in 1981, 1983, and 1992, respectively. In 1983, he joined Mitsubishi Electric Corporation, Hyogo, Japan. From 1992 to 1993, he was a visiting scientist in the Optoelectronics Computing Systems Center, University of Colorado at Boulder. In 1998, he joined Graduate School of Materials Science, Nara Institute of Science and Technology, Nara, Japan, as an associate professor. He was appointed professor in 2004. His current research interests are smart CMOS image sensors for biomedical applications and retinal prosthetic devices. He serves as an associate editor of IEEE Sensors Journal and is on the Editorial Board of Journal of Engineering, IET. He is a fellow of the Japan Society of Applied Physics and the Institute of Image, Information, and Television Engineers, and a senior member of IEEE.

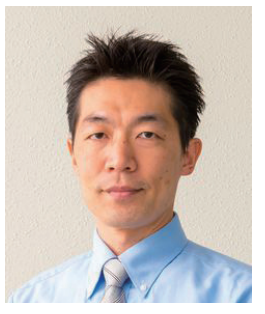

Takashi Tokuda received his B.E. and M.E. degrees in electronic engineering from Kyoto University, Kyoto, Japan, in 1993 and 1995, respectively. He received his Dr. Eng. degree in Materials Engineering from Kyoto University in 1998. He had been an assistant professor since 1999, and has been working as an associate professor since 2008 in the Graduate School of Materials Science, Nara Institute of Science and Technology. His research interests include CMOS image sensors, retinal prosthesis devices, bioimaging sensors, and biosensing devices.

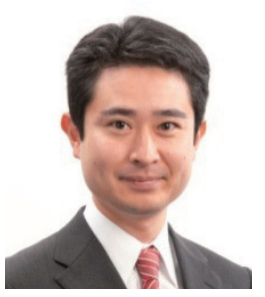

Toshihiko Noda received his B.E. and M.E. degrees in electrical and electronic engineering in 2001 and 2003 and a Ph.D. in engineering in 2006, all from the Toyohashi University of Technology (TUT), Aichi, Japan. He was a postdoctoral researcher at the Venture Business Laboratory in TUT from 2006 to 2007. He joined the faculty of the Intelligent Sensing System Research Center in TUT in 2008 as an assistant professor. Since 2009, he has been an assistant professor at the Nara Institute of Science and Technology, Nara, Japan. His current research focus is on retinal prosthesis devices and bioimaging with CMOS image sensors. 